\title{
ON APPLICATIONS OF SUMMARY EQUATION INDUCED BY QUADRILATERAL
}

\author{
F.N. GARIF'YANOV, E.V. STREZHNEVA
}

\begin{abstract}
Let $D$ be an arbitrary quadrilateral. On this quadrilateral, we consider a linear summary four-elements equation with the class of solutions holomorphic outside $D$ and vanishing at infinity. Their boundary the values satisfy the Hölder condition on each compact set containing no peaks. If the peaks are present, at them, at most logarithmic singularities are admitted. The free term is holomorphic on $D$ and its boundary value satisfies the Hölder condition. It is not assume to admit an analytic continuation through some segment of the boundary, that is, the solution and the free term belong to different classes of holomorphic functions. In order to regularize this equation on the boundary of the quadrilateral, we introduce a piece-wise linear Carleman translation mapping each side into itself by changing the orientation. This translation is discontinuous at the vertices and has fixed points at the centers of the side. The solution can be represented as a Cauchy type integral over a boundary with an unknown density invariant with respect to the shift on one pair of adjacent sides and anti-invariant on the other pair. We show that the regularization is equivalent. In some particular cases the obtained Fredholm equation is solvable. As an example, we choose an quadrilateral with a straight angle. We construct a system of entire functions of a completely regular growth biorthogonal to the system of powers with a piece-wise quasi-polynomial weight.
\end{abstract}

Keywords: equivalent regularization, biorthogonal systems of analytic functions, moment problem for entire functions of exponential type.

Mathematics Subject Classification: 30Exx: 30E05, 30E20, 30E25

\section{INTRODUCTION}

Let $D$ be a quadrilateral with vertices $t_{j}, j=\overline{1,4}$ and sides $\ell_{j}$ counted along the passing of a positive oriented boundary $\Gamma=\partial D\left(\left\{t_{1}, t_{2}\right\} \subset \bar{\ell}_{1}\right)$. The transformations

$$
\sigma_{j}(z)=t_{j}+t_{j+1}-z \quad\left(t_{5} \equiv t_{1}\right)
$$

maps $D$ into quadrilaterals having a joint side with it. We consider the equation

$$
(V f)(z) \equiv \sum_{j=1}^{4} \lambda_{j} f\left[\sigma_{j}(z)\right]=g(z), \quad z \in D,
$$

under the following assumptions:

1) $\lambda_{j} \neq 0$ for all $j$ and this is why we assume that $\lambda_{1}=1$.

2) A free term $g(z)$ is holomorphic in $D$ and its boundary value obeys $g^{+}(t) \in H(\Gamma)$.

3) A solution is sought in the class of the functions $f(z)$ holomorphic outside $D$ and decaying at infinity. Moreover, $f^{-}(t) \in H\left(\ell_{j}\right)$ for all $j$ and in the vertices, at most logarithmic singularities are admitted.

F.N. Garif'yanov, E.V. Strezhneva, On APPLICATIONS OF SUMmary EQUATION INDUCED By QUADRILATERAL.

(C) Garif'yanov F.N., Strezhneva E.V. 2019.

Submitted January 8, 2019. 
We denote such class of solutions by $B$. For more details of such choice we refer to the introduction in [1]. Here we only note that the non-triviality of equation (2) follows the nonconnectedness of the set $C \backslash \cup \sigma_{j}(D), j=\overline{1,4}$. The solution and the free term belong to different classes of holomorphic functions and the latter is not supposed to be analytically extended via some segment on the boundary.

First equation (2) was completely studied in paper [1] in a particular case, when $\lambda_{j}=(-1)^{j+1}$ and $D$ was an equilateral trapezium. Under the same choice of the coefficients, in paper [2], there was proposed a method of an equivalent regularization of equation (2) for an arbitrary quadrilateral and there was completely studied one more particular case, when $D$ is some quadrilateral, one of whose angles is straight. In paper [3], a regularization method was proposed for equation (2) as $\lambda_{j}=1$ for all $j$. In two aforementioned particular cases, a complete study of solvability was made. The comparison of the obtained results showed that the picture of solvability depends essentially on the choice of the coefficients. The aim of the present work is to study equation (2) in the case $\lambda_{1}=\lambda_{2}=1$ and $\lambda_{3}=\lambda_{4}=-1$ and to apply the obtained results to interpolation problems for entire functions of exponential type. The work consists of four sections.

The first section is an introduction. In the second section we make an equivalent regularization of equation (2). In the third section this equation is completely studied in the case, when $D$ is a quadrilateral with vertices $t_{1}=2^{-1}-i, t_{2}=2^{-1}, t_{3}=2^{-1}+i, t_{4}=-2^{-1}$ considered earlier in papers [2] and [3]. In the fourth section, equation (2) is interpreted in terms of entire functions of exponential type as a power Stieltjes moment problem on three rays with a piece-wise exponential weight. We construct biorthogonal on $\Gamma$ systems of analytic functions induced by this equation.

\section{Regularization of PROBlem}

We see a solution to equation (2) as a Cauchy type integral

$$
f(z)=\frac{1}{2 \pi i} \int_{\Gamma}(\tau-z)^{-1} \varphi(\tau) d \tau, \quad z \notin \bar{D}
$$

with an unknown density $\varphi \in H\left(\ell_{j}\right), j=\overline{1,4}$. It is clear that $\sigma_{j}(t): \vec{\ell}_{j} \rightarrow \vec{\ell}_{j}$ for all $j$ with a changing of the orientation, that is, a piece-wise linear function $\alpha(t)=\left\{\sigma_{j}(t), t \in \ell_{j}\right\}$ is an inverse Carleman shift on $\Gamma$ discontinuous at the vertices. The centers of these sides are fixed points of the shift.

We introduce a piece-wise constant function $\theta_{t}=\left\{1, t \in \ell_{1} \cup \ell_{2} ;-1, t \in \ell_{3} \cup \ell_{4}\right\}$ and an involutive operator $W: \varphi(t) \rightarrow \theta_{t} \varphi[\alpha(t)]$. We observe that the density in Cauchy type integral (3) is defined up to an analytically continuable in $D$ term $a^{+}(\tau)$. By choosing this term, without loss of generality we assume that

$$
W \varphi=\varphi .
$$

Indeed, we interpret relation (4) as a Carleman problem

$$
a^{+}-W a^{+}=W \varphi-\varphi .
$$

It is unconditionally solvable that is established by reducing it to the Riemann problem by the method of locally conformable gluing [4]. Then equation (2) is equivalent

$$
(A \varphi)(z) \equiv \frac{1}{2 \pi i} \int_{\Gamma} N(\tau+z) d \tau=g(z), \quad z \in D,
$$

where

$$
N(\beta)=\sum_{j=1}^{4} \lambda_{j}\left(\beta+a_{j}\right)^{-1}, \quad a_{j}=-\left(t_{j}+t_{j+1}\right)
$$


The following analogue of Sokhotski-Plemelj formula holds:

$$
\left(A^{+} \phi\right)(t)=-2^{-1}(W \varphi)(t)+(A \varphi)(t), \quad t \in \Gamma,
$$

and a special integral operator operator $(A \varphi)(t)$ is obtained by the formal replacing $z \in D$ by $t \in \Gamma$ and is treated in the sense of the Cauchy principal value. We apply the operator $W$ to the both sides of identity (6) and in the special integral, we replace the variable $\tau$ by $\alpha(\tau)$ taking into consideration (4), the change of the orientation and the condition $\alpha^{\prime}(\tau)=-1, \tau \neq t_{j}$. Then we sum up the obtained relation with the initial one and we arrive at the equation

$$
T \varphi=g^{+}+W g^{+},
$$

where $T=-I+A+W A W$, and $I$ is the identity mapping. We introduce a Banach space $\widetilde{C}(\Gamma)$, which is the set of the functions $\varphi(t) \in C\left(\bar{\ell}_{j}\right), j=\overline{1,4}$ equipped with the norm

$$
M=\max |\varphi(t)|, \quad t \in \Gamma .
$$

Lemma 1. The operators $A$ and $W$ anticommute in $\widetilde{C}(\Gamma)$ up to a compact term, that is, equation (7) is second Fredholm integral equation.

The proof is reduced to a straightforward checking of the boundedness of the kernel

$$
K(t, \tau)=N(\tau+t)+\theta_{\tau} \theta_{t} N[\alpha(\tau)+\alpha(t)]
$$

for various options of mutual location of the points $\tau$ and $t$ on the sides of $\Gamma$.

Theorem 1. Equation (2) possesses at most finitely many solvability conditions being the solvability conditions of equation (7).

Proof. Once equation (7) is solvable, it necessarily has a solution with property (4), see [5] for details. Then equation (7) implies equation (2) since by the mentioned principle of locally conformal gluing, a homogeneous Carleman problem $a^{+}=-W a^{+}$possesses a trivial solution only.

Remark 1. The considered set of the coefficients in equation (2) is the last among three possible options allowing one to regularize this equation by the method proposed in [1]. The case $\lambda_{1}=\lambda_{4}=1, \lambda_{2}=\lambda_{3}=-1$ has no essential differences.

We consider a corresponding homogeneous equation

$$
T \varphi=0 .
$$

We can choose its fundamental system of solutions so that each involved function satisfies either (4) or an opposite condition

$$
\varphi=-W \varphi
$$

see [5].

It is clear that $K(t, \tau)=K(\tau, t)$. The functions with property (11) are immediately orthogonal to the right hand side in equation $(7)$.

Indeed, the identity $b(t)=-b[\alpha(t)]$ implies that $\int_{\Gamma} b(t) d t=0$.

\section{Complete Studying of A PARTicular CASE}

Let $D$ be a quadrilateral with the vertices at $t_{1}=2^{-1}-i, t_{2}=2^{-1}, t_{3}=2^{-1}+i, t_{4}=-2^{-1}$.

Lemma 2. The fundamental system of solutions of (10) contains no functions with property (4). 
Proof. We argue by contradiction and assume the opposite. Let us estimate the absolute value of the integral term in equation (10). In view of the symmetry of $\Gamma$ and the choice of the coefficients in problem (2), it is sufficient to consider two cases only.

I. Identity (8) is attained at $t \in \ell_{1}$; in this case $\alpha(t)=1-i-t, \theta_{t}=1$. For the sake of brevity, we introduce the notations $u=\tau+t$ and $b_{j}(t)=\left|\int_{\ell_{j}} \varphi(\tau) K(t, \tau) d \tau\right|$. The following four subcases are possible:

a) $\tau \in \ell_{1}$ and then $\alpha(\tau)=1-i-\tau, \theta_{\tau}=1$ and

$$
K(t, \tau)=(u-1-i)^{-1}-(u+i)^{-1}-(u-i)^{-1}-(u-1+3 i)^{-1}+(u-2+3 i)^{-1}
$$

and then $b_{1}(t)<2.41 M$.

b) $\tau \in \ell_{2}$ and then $\alpha(\tau)=1+i-\tau, \theta_{\tau}=1$ and

$$
K(t, \tau)=(u-2-i)^{-1}+(u-2+i)^{-1}-(u+i)^{-1}-(u-i)^{-1}
$$

and then $b_{2}(t)<2.42 M$.

c) $\tau \in \ell_{3}$ and then $\alpha(\tau)=i-\tau, \theta_{\tau}=-1$ and $K(t, \tau)=0$.

d) $\tau \in \ell_{4}$ and then $\alpha(\tau)=-i-\tau, \theta_{\tau}=-1$ and

$$
K(t, \tau)=(u-1-i)^{-1}-(u-i)^{-1}+(u+3 i)^{-1}-(u-1+3 i)^{-1}
$$

and then $b_{4}(t)<0.67 \sqrt{2} M$.

Since $2.41+2.42+0.67 \sqrt{2}<2 \pi$, then $\varphi \equiv 0$.

II. Identity (8) is attained at $t \in \ell_{3}$; in this case $\alpha(t)=i-t, \theta_{t}=-1$. The following four subcases are possible:

a) $\tau \in \ell_{1}$ and then $\alpha(\tau)=1-i-\tau, \theta_{\tau}=1$ and $K(t, \tau)=0$.

b) $\tau \in \ell_{2}$ and then $\alpha(\tau)=1+i-\tau, \theta_{\tau}=1$ and

$$
K(t, \tau)=(u-1+i)^{-1}-(u+i)^{-1}+(u-3 i)^{-1}-(u-3 i-1)^{-1}
$$

and then $b_{2}(t)<0.67 M$.

c) $\tau \in \ell_{3}$ and then $\alpha(\tau)=i-\tau, \theta_{\tau}=-1$ and

$K(t, \tau)=(u-1+i)^{-1}+(u-1-i)^{-1}-(u+i)^{-1}-(u+1-3 i)^{-1}-(u+1-i)^{-1}+(u-3 i)^{-1}$

and then $b_{3}(t)<2.41 \sqrt{2} M$.

d) $\tau \in \ell_{4}$ and then $\alpha(\tau)=-i-\tau, \theta_{\tau}=-1$ and

$$
K(t, \tau)=(u-1+i)^{-1}+(u-1-i)^{-1}-(u+1-i)^{-1}-(u+1+i)^{-1}
$$

and then $b_{4}(t)<2.46 \sqrt{2} M$.

The latter estimate does not allow us to prove the lemma and this is why we employ condition (4). In Subcase d), instead of kernel (9), we introduce a new kernel

$$
K_{1}(t, \tau)=2^{-1}(K(t, \tau)-K(t,-i-\tau))
$$

and then

$$
\left|\int_{\ell_{4}} \varphi(\tau) K_{1}(t, \tau) d \tau\right| \leq 0.8 \sqrt{2} M .
$$

Since $0.67+2.41 \sqrt{2}+0.8 \sqrt{2}<2 \pi$, we get $\varphi \equiv 0$ and this completes the proof.

Corollary 1. Fundamental system of solutions of equation (10) can contain only functions with property (11).

Theorem 2. The homogeneous equation $(V f)(z)=0, z \in D$, possesses only the trivial solution. The inhomogeneous equation (2) is unconditionally solvable and possesses a unique solution. 
Remark 2. It could seems that Theorem 2 remains true also in the case $\lambda_{1}=\lambda_{4}=1$, $\lambda_{2}=\lambda_{3}=-1$. Here the fundamental system of solutions of the corresponding homogeneous Fredholm equation is empty. We do not provide the proof since it is very bulky and at the same time, this property is not employed below.

\section{Applications to MOMEnT PROBLEM}

We proceed to applications of equation (2). We interpret the set $D$ as an adjoint indicator diagram of an entire function of exponential type $F(z)$, an upper function, associated in the Borel sense [6, Sect. 1.1] with a lower function $f(z) \in B$. Then equation (2) is equivalent to

$$
\sum_{j=1}^{2} \int_{0}^{\infty} F(x) \exp \left(-x \sigma_{j}(z)\right) d x-\sum_{j=3}^{4} \int_{L_{j}} F(\tau) \exp \left(-\tau \sigma_{j}(z)\right) d \tau=g(z), \quad z \in D
$$

where $L_{3}$ is the ray $\arg \tau=5 \pi / 4, L_{4}$ is the $\operatorname{ray} \arg \tau=3 \pi / 4$. Equating the Maclaurin coefficients in the left hand side and the right hand side of relation (12), we get:

$$
\begin{aligned}
& E\left[F(t), t^{k}\right] \equiv \sum_{j=1}^{2} \int_{0}^{\infty} F(x) x^{k} \exp \left(a_{j} t\right) d t-\sum_{j=3}^{4} \int_{L_{j}} F(t) t^{k} \exp \left(a_{j} t\right) d t=g^{(k)}(0), \\
& a_{j}=-\left(t_{j}+t_{j+1}\right), \quad k=\overline{0, \infty} .
\end{aligned}
$$

The latter problem can be regarded as a generalization of known Stieltjes and Hamburger moment problem for the case of three rays with a piece-wise polynomial weight.

Remark 3. The case when the adjoint indicator diagram of an exponential function of exponential type $F(z)$ is some smaller convex set $D_{1} \subset D$ is possible but is not of interest. In this case relation (2) holds not only as $z \in D$ but also in the vicinity of the infinity, that is, it is overdetermined. To satisfy this relation, it is necessary but not sufficient that the free term to be analytically continuable through some segment $\Gamma$ into the vicinity of the infinity and $g(\infty)=0$. For more details we refer to Remark 1 in [2].

Theorem 3. A power moment problem $E\left[F(t), t^{k}\right]=\beta_{k}, k=\overline{0, \infty}$ is unconditionally solvable in the class of entire functions of exponential type $F(z)$ associated in the Borel sense with a lower function $f(z) \in B$ and possesses a unique solution under the condition that the sum of the series

$$
g(z)=\sum_{k=0}^{\infty} \frac{\beta_{k} z^{k}}{k !}
$$

is holomorphic in $D$ and $g^{+}(t) \in H(\Gamma)$.

Remark 4. The assumptions of the theorem hold apriori if the convergence radius of power seres (13) satisfies $R>\sqrt{5} / 2$; this can be checked easily.

Let $g_{m}(z)=z^{m} / m$ !. We consider the system of the functions

$$
\left\{f_{m}(z)\right\}:\left(V f_{m}\right)(z)=g_{m}(z), \quad z \in D, \quad m=\overline{0, \infty} .
$$

The corresponding system of upper functions $\left\{F_{m}(z)\right\}$ satisfies the conditions $E\left[F_{m}(t), t^{k}\right]=$ $\delta_{m, k}$, that is, it is biorthogonal to the system of powers on three rays with some piece-wise quasi-polynomial weight. Similar to [2], one can show that this system of entire functions of a completely regular growth [6, Ch. III] with a piece-wise trigonometric indicator

$$
h(\theta)=\left\{2^{-1} \cos \theta+\sin \theta, \theta \in[0,3 \pi / 4] ;-2^{-1} \cos \theta, \theta \in[3 \pi / 4, \pi]\right\}
$$

and $h(\theta)=h(-\theta)$. 
Now we consider the system of functions with property (4):

$$
\left\{\varphi_{m}(\tau)\right\}:\left(A \varphi_{m}\right)(z)=(-1)^{m} g_{m}(z), \quad m=\overline{0, \infty} ; \quad z \in D
$$

The definition of this system implies immediately that

$$
\frac{1}{2 \pi i} \int_{\Gamma} \varphi_{m}(\tau) N^{(k)}(\tau) d \tau=\delta_{m, k} .
$$

System (15) is inconvenient since the densities $\varphi_{m}(\tau)$ can be not boundary values of functions analytic outside $\bar{D}$ and decaying at infinity. This is why we rewrite (16) as

$$
-\frac{1}{2 \pi i} \int_{\Gamma} f_{m}^{-}(\tau) N^{(k)}(\tau) d \tau=\delta_{m, k},
$$

where $f_{m}(z)$ is Cauchy type integral (3) with density $\varphi_{m}(\tau)$.

Functions (14) are formally defined outside $\bar{D}$. In fact, they can be analytically continued inside $D$ through each its side. At that, the vertices are the branching points. We introduce two triangles: $\Delta_{1}$ with vertices at $t_{1}, t_{2}, t_{4}$ and $\Delta_{2}$ with vertices $t_{2}, t_{3}, t_{4}$. We define functions (14) in $\Delta_{1}$ via the analytic continuation through the side $\ell_{1}$, that is,

$$
f(z)=f_{m}(z+2 i-1)+f_{m}(z-1)-f_{m}(z+2 i)-\frac{(1-i-z)^{m}}{m !}, \quad z \in \Delta_{1} .
$$

In the other triangle, we define functions (14) by analytic continuation through the side $\ell_{3}$, that is,

$$
f(z)=f(z+1-2 i)+f(z+1)-f(z-2 i)-\frac{(i-z)^{m}}{m !}, \quad z \in \Delta_{2} .
$$

Thus, functions (14) are holomorphic in the plane with a two-sided cut along the polyline with vertices at $t_{1}, t_{4}, t_{2}, t_{3}$ counted in the order of location along the polyline.

\section{BIBLIOGRAPHY}

1. F.N. Garif'yanov, S.A. Modina. On the four-element equation for the functions analytic beyond a trapezoid and its applications // Sibir. Matem. Zhurn. 52:2, 243-249 (2011). [Siber. Math. J., 52:2, 191-196 (2011).]

2. F.N. Garif'yanov. Summary equation for functions analytical outside a quadrangle // Izv. VUZov. Matem. 10, 3-7 (2016). [Russ. Math. (Iz. VUZ). 60:10, 1-4 (2016).]

3. F.N. Garif'yanov. On summary equation generated by a quadrangle // Izv. VUZov. Matem. 1, 27-33 (2018). [Russ. Math. Iz. VUZ. 62:1, 23-28 (2018).]

4. Eh. I. Zverovich. A method of locally conformal gluing // Dokl. AN SSSR. 205:4, 767-770 (1972). [Sov. Math. Dokl. 13, 1003-1007 (1972).]

5. E.P. Aksent'eva, F.N. Garif'yanov. On the investigation of an integral equation with a Carleman kernel // Izv. VUZov. Matem. 4, 43-51 (1983). [Soviet Math. Iz. VUZ. 27:4, 53-63 (1983).]

6. L. Bieberbach. Analytische Fortsetzung. Sringer, Berlin (1955). (in German).

Farkhat Nurgayazovich Garif'yanov

Kazan State Power Engineering University

Krasnosel'skaya str. 51

420066, Kazan, Russia

E-mail: f.garifyanov@mail.ru

Elena Vasil'evna Strezhneva,

Kazan National Research Technical

University named after A. N. Tupolev - KAI

Karl Marx str. 10

420111, Kazan, Russia

E-mail: strezh@yandex.ru 